\title{
Topological approach to atomic charges, electric moments, axial vectors, spontaneous polarization and optical rotation in $\alpha-\mathrm{LiIO}_{3}$ at $296 \mathrm{~K}$.
}

\author{
M. Karppinen \\ Institute of Chemistry, University of Uppsala, Box 531, Uppsala S-75121 Sweden. \\ Correspondence e-mail: markkuk96@gmail.com
}

\begin{abstract}
Optical rotation and spontaneous polarization are determined from the point charge model in four structure descriptions of a chiral ionic solid $\alpha-\mathrm{LiIO}_{3}$ crystal. Net atomic charges of I and $\mathrm{O}$ atoms are iterated under ionic constraints. The inverted ratio of the second electric moments, derived from the polar vectors of second rank in the semi-axis directions of the quadric around the iodate ion, is fitted equal to the ratio of the optical refractive indices in the semi-axis directions of the indicatrix. The principal refractive indices contain information about the axial vectors of second rank, which are formed through vector cross products between a wave vector in the propagation direction of light and the second electric moments acting in the two other semi-axis directions of the quadric. The calculated axial vectors reveal the magnitude of rotation and the handedness of the dominant vector of them gives the sense of optical rotation character of the crystal. They are converted to the principal gyration tensor components and the sense and magnitude of optical rotation is determined in the direction of the optic axis. The sense and magnitude of the spontaneous polarization vectors are computed in the four structure
\end{abstract}


alternatives. The enantiomorphic pairs are identified amongst the four structure descriptions and the absolute crystal structure of $\alpha-\mathrm{LiIO}_{3}$ is discussed.

Keywords: LiIO3, atomic charges, electric moments, polar and axial vectors spontaneous polarization,optical rotation

\section{Introduction}

The origin of some physical properties in crystals is described to be closely associated with a point particle model. Dielectric properties such as pyroelectricity, piezoelectricity and ferroelectricity are found to be very structure sensitive and even optical rotation has been related to the atomic arrangement in crystals. The aim of this paper is to describe the distribution of accurate charge densities in an ionic solid. A system of point charges creates an electric field and the electric potential arising from it at any point outside the system can be developed to a set of electric multipole moments (zeroth, first, second, third etc.). These moments can be related to the measured physical properties in crystals.

Within the 11 pairs of enantiomorphic space groups exist four pairs, in which the screw rotations are chiral and which can be easily differentiated as the right- and left-handed enantiomorphic pairs by inversion. Three screw rotations $2_{1}, 4_{2}$, and $6_{3}$ are invariant in respect to inversion. They are regarded as achiral, though the atomic arrangements in the space groups they exist are chiral. [1] Hence in some crystals with achiral screw axes, which both are chiral and polar and have specific atomic arrangements, the absolute configuration of the enantiomorphic pairs can be hard to solve. Experimental tests must be carried out in order to make the correct decision about the sense of structural chirality. The sense of optical chirality is always in some way connected to the sense of structural chirality. One example of this kind of puzzling crystal is $\alpha-\mathrm{LiIO}_{3}$, which is both polar and optically active and experimentally tested by several research groups, is taken under study in this paper.

The crystal structure of $\alpha-\mathrm{LiIO}_{3}$ was first determined with X-ray technique in the space group $P_{6}$ by Rosenzweig and Morosin. [2] Morphologically steep hexagonal pyramids of $\alpha$ - $\mathrm{LiIO}_{3}$ crystals developed a positive polarity at a larger end of the crystal in a positive $c$-axis direction upon compression. The crystal structure established that the apices of the iodate ions pointed in 
a negative $c$-axis direction. The results of this piezoelectric test were related in a private communication to the structure, where a positive charge was developed on the face $\{00.1\}$ on expansion $(=$ extension $=$ tensile stress $)$, towards which also the apices of the $\mathrm{IO}_{3}{ }^{-}$ions were declared to point [3] in contrast to the first determination. [2]

The first study of the absolute configurations of the two enantiomorphic structures of $\alpha$ - $\mathrm{LiIO}_{3}$ by anomalous X-ray scattering and by the morphology of the crystal led to the structures as presented in Figs. 1(a) and 1(b), respectively, but with opposite optical chiralities. [4] More explicit explorations by neutron and anomalous X-ray scattering at several temperatures resulted in two structural configurations. [5] In a levorotatory description the apices of the iodate ions were described to point to a positive and in a dextrorotatory configuration, with the reversed set of z-coordinates, to a negative c-axis direction, respectively. The same assignment of the absolute configurations was made as in the first study of optical chirality [4] without the examination of the sign of optical rotation in the crystal.

In another work a clear dextrorotatory Airy's spiral was observed under a conoscopic illumination in slices, cut perpendicular to the unique $c$-axis from a large crystal of $\alpha-\mathrm{LiIO}_{3}$ of high optical quality. [6] The collected set of anomalous X-ray intensities of Bijvoet pairs with the Miller indices $h k l$ and $h k l$ from a spherical dextrorotatory crystal and the comparison of the differences between the observed and calculated structure factors according to the models presented in the earlier study [5] verified the agreement between a dextrorotatory configuration and a model with the apices of iodate ions pointing to a positive c-axis direction without to test the sense of polarization in this crystal. [6] The result concerning optical chirality became discrepant from the earlier explored models. [4,5] Four possible structure descriptions were presented for $\alpha-\mathrm{LiIO}_{3}$, each with an optical rotation extracted from the I-O-I-O helices. They are shown in the projection views on (001) in Figs. 1(a)-(d). [6] The structure alternatives are left-handed in Figs. 1(a) and 1(c) and right-handed in Figs. 1(b) and 1(d). The structures in Figs. 1(a) and 1(b) are related with a (001) mirror operation to each other, which changes the direction of the polar groups and both the structural and optical chirality between these two structure descriptions. The alternative in Fig. 1(c) has the same structural and optical chirality as the model in Fig. 1(a) but with an opposite direction of the polar groups, which makes the structures in Figs. 1(b) and 1(c) mirror images with the opposite handedness and optical chirality. 


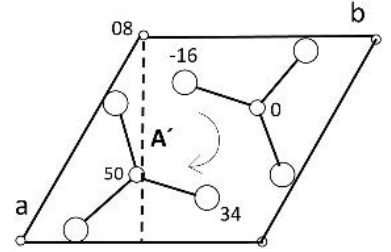

1(a)

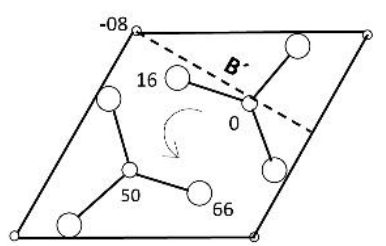

1(b)

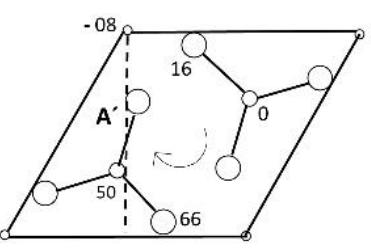

1(c)

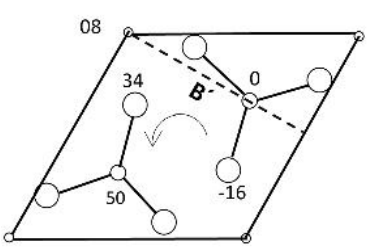

1(d)

Figure. 1. Projections of four $\alpha-\mathrm{LiIO}_{3}$ structure views on (001) planes (from [6] and [11]. Small circles Li, medium I and large O. Fractions of atomic z-coordinates (x 100). Both of the two pairs (a)-(c) and (b)-(d) have the same structural and optical chirality but opposite direction of the polar groups. Both of the two pairs (a)-(b) and (c)-(d) have opposite structural and optical chirality. The projections are displayed in the right-hand coordinate system of axes as shown in Fig.1(a). Broken lines, denoted by $A^{\prime}$ and B', are the orientations of the dominant axial vectors.

In 10 of 21 non-centrosymmetric classes the crystals are expanding on heating and can produce electric polarization in polar directions, which can be detected in a pyroelectric measurement. When a crystal is mechanically drawn out and extended by an increasing tension, it develops an electric polarization with the same sense and at the same ends of the crystal as in a pyroelectric measurement. The sign of polarization produced by a tensile stress is reversed by an application of compressive stress on the piezoelectric crystals. The release of compressive stress causes a measurable tensile stress in crystals. [7] Hence the piezo- and the pyroelectric coefficients are given with the same sign.

Three independent works about the correlation of the crystal structure with piezo- and pyroelectric coefficients of $\alpha-\mathrm{LiIO}_{3}$ have been reported. In the first measurement the apices of iodate ions were denoted to point to the face $\{00.1\}$, which developed a positive charge both on extension and heating. [8] In the second study [9] the absolute crystal structure of $\alpha-\mathrm{LiIO}_{3}$ was determined with anomalous X-ray scattering resulting in the same atomic arrangement as in the first structural study. [2] The absolute structure was ruled out, because the choice of Miller indices led to a negative piezoelectric coefficient, $d_{33}<0$. The indices were changed from $h k l$ to $\hbar \hbar l$ and the atomic coordinates from $x y z$ to $\bar{x} \bar{y} z$ in order to be consistent with the other determination. [8] The structural results [9] became now in accordance with the piezoelectric IEEE Standard convention, according to which the positive sense of polarization may be taken to coincide with the positive axial sense determined by piezoelectric standards. [10] Following this convention the piezoelectric and pyroelectric coefficients, $d_{33}$ and $p_{3}$, and the spontaneous 
polarization vector, $\boldsymbol{P}_{\boldsymbol{s}}$, will always be positive in polar crystals with no attention paid to the absolute structure.

In the third work the absolute configuration, polarity, morphology and optical activity of $\alpha$ $\mathrm{LiIO}_{3}$ crystal were explored. A number of slices cut perpendicular to the polar axis of both dextro- and levorotatory crystals were studied under a conoscopic illumination. [11] The structural parameters were taken from the first structure determination [2] with the tapered end of the crystal as $+c$-axis direction, where the apices of iodate groups were also assigned to point. A negative charge was observed to develop in the $+c$-axis direction both in dextro- and levorotatory crystals on extension. The conclusion was that both $d_{33}$ and $p_{3}$ are negative in the $\alpha-\mathrm{LiIO}_{3}$ crystal, which made the results in the earlier studies disputed. ${ }^{4,5}$ However, the assignments of the absolute configurations for both the dextro- and levorotatory enantiomorphs, in Figs. 1(a) and 1(d), respectively, were reported to be consistent with the research results in [6].

The presented studies of the structure-property relationships above show divergent results. It is not clarified which two of the four structure descriptions are the actually existing enantiomorphic pairs. Partly for these reasons $\alpha-\mathrm{LiIO}_{3}$ was taken under study.

This paper presents an alternative way to calculate electric polarization and optical rotation from the accurately determined point charge model in a simple ionic solid. In this work net atomic charges in the iodate ion, $\mathrm{IO}_{3}{ }^{-}$, are iterated, where the total ionic charge of -1 is distributed between iodine and three symmetry related oxygen atoms. This type of treatment offers an excellent opportunity to clarify, whether suitable parameters can be obtained for the theoretical description of some physical properties associated with the $\alpha-\mathrm{LiIO}_{3}$ crystal. Concerning the optical rotation this study deviates from the early "Coupled oscillator" theories applied in the electromagnetic field $[12,13]$ and from the later developed "electronic polarizability" theories $[6,14,15]$ with oscillating dipoles, caused by an electromagnetic wave of light on the valence electrons of the atoms. Those theories have a high complexity with too many unknown variables. This work relies on the fact that the polar vectors of second rank, derived from the point charge model, interact with light and generate axial vectors of second rank. The axial vectors with the distinct length and handedness reveal both the magnitude and the sense of the rotational character of the crystal. The magnitudes of principal gyration tensor components, $g_{11}=g_{22} \neq g_{33}$, are defined and the sense of optical rotation is determined in the 
positive $c$-axis direction in the four structure descriptions of $\alpha-\mathrm{LiIO}_{3}$. Spontaneous polarization and the absolute crystal structure are discussed and the enantiomorphic pairs of $\alpha-\mathrm{LiO}_{3}$ are presented.

\section{Structural and theoretical basis}

All transformed and converted atomic positional coordinates in this study are taken from the right-handed absolute structure description of $\alpha-\mathrm{LiIO}_{3}$ according to Fig. 1(b). [5] It crystallizes in a hexagonal space group $P 6_{3}$. It is optically negative crystal, $n_{o}>n_{e}$, with two symmetry related iodate ions in the unit cell. It can be assumed that the correct determination of absolute polarity and optical chirality will be easier, if the atomic net charges are known. $\alpha-\mathrm{LiIO}_{3}$ is an insulator, an ionic solid, where the rule of integer charges is not broken. It is pyro- and piezobut not ferroelectric crystal and has a spontaneous polarization vector, $\boldsymbol{P}_{\boldsymbol{s}}$, which can't be measured, but its value can be calculated when the net atomic charges are known. $\mathrm{Li}^{+}$ion has lost its valence electron and the two core electrons of it orbit closely around the positively charged nucleus making it a spherical particle, which does not contribute in any way to the distribution of electric moments in the crystal. The multinuclear negative particles, as $\mathrm{IO}_{3}{ }^{-}$ion, have several polarizable valence electrons, which the atoms can deal in the covalent bonds. An anisotropic distribution of the valence electrons gives electric net charges for the atoms and makes the $\mathrm{IO}_{3}{ }^{-}$ion polar. Point charges generate a series of electric multipole moments with the variable symmetry dependent magnitudes in the different directions of the crystal structure. When a light front is travelling through a crystal, it interacts with the electric moments and forms an indicatrix, which is a quadric (= a surface of second rank). It can be described by the equation of an ellipsoid of revolution about the unique symmetry axis in tetragonal, hexagonal and trigonal crystals with the mutually perpendicular semi-axes, which are accurately measurable quadratic refractive indices, $n_{o}, n_{o}, n_{e}$. Equally to the indicatrix, the tetragonal, hexagonal and trigonal crystals have another quadric, which is an ellipsoidal representation surface with polar quadratic second electric moments as the mutually orthogonal semi-axes. The second electric moments, either in the principal $a$ - or $b$-axis directions, are multiplied by $\cos ^{2} 30^{\circ}$-term in calculations to get them to line parallel with the semi-axes in both quadrics in hexagonal and trigonal crystals. The refractive indices are constants at $296 \mathrm{~K}$ and the corrected second electric moments, $\Theta_{x x}^{\prime}, \Theta_{y y}^{\prime}$ and $\Theta_{z z}^{\prime}$, contain the sum of the products of the constant 
atomic coordinates and charges, of which the charges are sole variables with the mutual correlation and the dependence on the total ionic charge of the $\mathrm{IO}_{3}{ }^{-}$ion. The second electric moments are polar vectors of second rank, which have even parity and are positive in both directions in the axis system of the quadric. One of the surfaces is a positive and another one a negative ellipsoid of revolution.

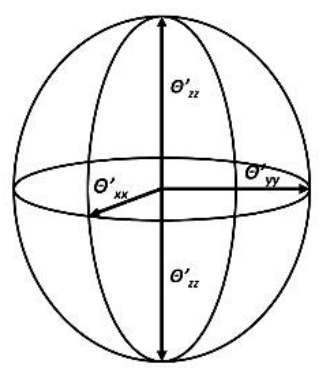

2(a)

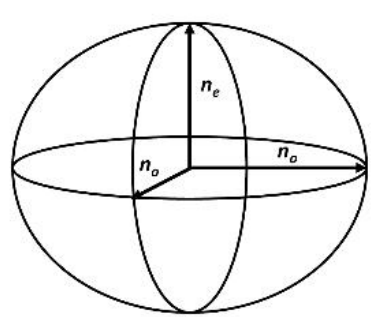

2(b)

Figure 2. Quadrics (not scaled) of (a) the polar second electric moments and (b) the optical indicatrix of $\alpha$-LiIO3.

The both surfaces as illustrated in Figs. 2(a) and 2(b), (not scaled), are defined with different techniques from the $\alpha-\mathrm{LiIO}_{3}$ crystal, which make them interesting objects for an equivalent topological study (Encyclopedia Britannica). The iodate ion, $\mathrm{IO}_{3}{ }^{-}$, has a pyramidal structure with three symmetry related oxygen atoms. It has a polar vector in the principal $c$-axis direction of the crystal and its asymmetry contributes to the electric and optical properties of the crystal. Net atomic charges of the iodine and oxygen atoms are iterated until the inverted ratio of the compilated second electric moments reaches the value of the corresponding ratio of refractive indices, which is used as a reference.

The lattice parameters and the atomic position coordinates of $\mathrm{Li}, \mathrm{I}$ and $\mathrm{O}(1)$ are taken from the study by anomalous X-ray scattering [5], which are regarded [16] as most precise structural parameters of $\alpha-\mathrm{LiIO}_{3}$ crystal, together with the coordinates of $\mathrm{O}(2)$ and $\mathrm{O}(3)$, derived from the symmetry cards; $X, Y, Z,-Y, X-Y, Z, Y-X,-X, Z,-X,-Y, 1 / 2+Z, Y, Y-X, 1 / 2+Z, X-Y, X, 1 / 2+Z$, for the absolute structure description in Fig.1(b) stand in TABLE I. The atoms of one $\mathrm{IO}_{3}{ }^{-}$ion in each unit cell of the four structure alternatives are translated to middle of the unit cell in $a$ and $b$-axis directions with the iodine atoms to the coordinates $\mathrm{x}=\mathrm{y}=1 / 2$. The atoms are given the initial charges of -0.25 and the $\mathrm{z}$-coordinates are transformed in a following way: $-0.25 \mathrm{x}$ $0.5-0.25 \times 3 \times 0.6618=-0.62135$, with the center of charge at $\mathrm{z}=-0.62135 /-1=0.62135$. The 
center of charge in iodate ion is moved to $\mathrm{z}=1 / 2$ as follows: $0.62135-0.5=0.12135$ that gives $\mathrm{z}=0.5-0.12135=0.37865$ for the iodine and $\mathrm{z}=0.6618-0.12135 / 3=0.54045$ for oxygens in Figs. 1(b) and (c). The structures in Figs. 1(a) and (d) have the opposite direction of the polar groups with the reversed $\mathrm{z}$-coordinates and the transformed coordinates there will be $\mathrm{z}=$ 0.62135 for iodine and $z=0.45955$ for oxygens. One iodate ion is standing now in the middle of each unit cell in the four structure descriptions with the centers of charges at the coordinates $\mathrm{x}=\mathrm{y}=\mathrm{z}=1 / 2$ in order to make the calculations more convenient. $\mathrm{Li}^{+}$ions are omitted from the calculations. The transformed positional parameters in the four structure descriptions make the iterated parameters of the second electric moments comparable with each other and they are presented in TABLE II.

TABLE I. Lattice parameters and the position coordinates of $\mathrm{Li}$, I and $\mathrm{O}(1)$ together with the coordinates of $O(2)$ and $O(3)$.

\begin{tabular}{|c|c|c|c|}
\hline \multicolumn{2}{|c|}{$a=b=5.4818 \AA$} & \multicolumn{2}{c|}{$c=5.1725 \AA$} \\
\hline Atom & $\mathrm{x}$ & $\mathrm{y}$ & $\mathrm{z}$ \\
\hline $\mathrm{Li}$ & 0 & $2 / 3$ & -0.0822 \\
\hline $\mathrm{I}$ & $1 / 3$ & 0.3426 & 0 \\
\hline $\mathrm{O}(1)$ & 0.2479 & 0.9053 & 0.1618 \\
\hline $\mathrm{O}(2)$ & 0.6574 & 0.7521 & " \\
\hline $\mathrm{O}(3)$ & 0.0947 & & \\
\hline
\end{tabular}

Table II. Transformed position parameters of $\mathrm{IO}_{3}{ }^{-}$-ions.

\begin{tabular}{|c|c|c|c|c|}
\hline Figure & Atom & $\mathrm{x}$ & $\mathrm{y}$ & $\mathrm{z}$ \\
\hline $1(\mathrm{a})$ & $\mathrm{I}$ & 0.5 & 0.5 & 0.62135 \\
\hline & $\mathrm{O}(1)$ & 0.414567 & 0.175933 & 0.45955 \\
\hline & $\mathrm{O}(2)$ & 0.824067 & 0.738633 & “ \\
\hline & $\mathrm{O}(3)$ & 0.261367 & 0.585433 & " \\
\hline $1(\mathrm{~b})$ & $\mathrm{I}$ & 0.5 & 0.5 & 0.37865 \\
\hline & $\mathrm{O}(1)$ & 0.414567 & 0.175933 & 0.54045 \\
\hline & $\mathrm{O}(2)$ & 0.824067 & 0.738633 & " \\
\hline & $\mathrm{O}(3)$ & 0.261367 & 0.585433 & " \\
\hline $1(\mathrm{c})$ & $\mathrm{I}$ & 0.5 & 0.5 & 0.37865 \\
\hline
\end{tabular}




\begin{tabular}{|c|c|c|c|c|}
\hline & $\mathrm{O}(1)$ & 0.824067 & 0.585433 & 0.54045 \\
\hline & $\mathrm{O}(2)$ & 0.261367 & 0.175933 & “ \\
\hline & $\mathrm{O}(3)$ & 0.414567 & 0.738633 & " \\
\hline $1(\mathrm{~d})$ & $\mathrm{I}$ & 0.5 & 0.5 & 0.62135 \\
\hline & $\mathrm{O}(1)$ & 0.824067 & 0.585433 & 0.45955 \\
\hline & $\mathrm{O}(2)$ & 0.261367 & 0.175933 & “ \\
\hline & $\mathrm{O}(3)$ & 0.414567 & 0.738633 & " \\
\hline
\end{tabular}

\section{Iteration of net atomic charges and calculation of second electric moments}

Equations (1) and (2) are presented in the study of $\mathrm{LiNaSO}_{4}$ [17] and they are previously discussed in $[18,19]$. Equations (3)-(5) will give the net values of the polar second electric moments. $X, Y$ and $Z$ are the reference coordinates of the common center of electric moments $(=1 / 2)$.

$$
\begin{gathered}
\varphi=\Sigma\left(e_{i}\right) / R+\Sigma\left(e_{i} z_{i}\right) / R^{2}+\Sigma\left(e_{i} z_{i}^{2}\right) / R^{3}+\Sigma\left(e_{i} z_{i}^{3}\right) / R^{4}+\ldots \\
\mu_{z}^{\prime}=\Sigma e_{i}\left(z_{i}-Z\right)=\mu_{z}-q Z \\
\Theta_{z z}^{\prime}=\Sigma e_{i}\left(z_{i}{ }^{2}-Z^{2}\right) \cdot c^{2} \\
\Theta_{x x}^{\prime}=\Sigma e_{i}\left(x_{i}{ }^{2}-X^{2}\right) \cdot \cos ^{2}(30) \cdot a^{2} \\
\Theta_{y y}^{\prime}=\Sigma e_{i}\left(y_{i}{ }^{2}-Y^{2}\right) \cdot \cos ^{2}(30) \cdot b^{2}
\end{gathered}
$$

Eq. (1) is an expression for the total electrostatic potential, $\varphi=\Sigma\left(e_{i}\right) /(R / 2)$, of the system of point charges, which must be fulfilled in ionic solids. $\varphi$ is originally put forward in a polar coordinate system and developed into a power series in a Cartesian coordinate system as it is standing in the Eq. (1) $[18,19]$.

It contains the components of the zeroth, first, second, third etc. electric moments in the principal axis direction of the system of point charges. It is applied only in the polar $c$-axis direction of $\alpha-\mathrm{LiIO}_{3}$ to iterate the final z-coordinates for iodine and oxygen atoms. When the power series is truncated after the fourth term, it gives the accurate z-coordinates for iodine and oxygens in the iteration. Input of net atomic charges, $\Sigma\left(e_{i}\right)=-1, \mathrm{R}=1$ and the z-coordinates of I and $\mathrm{O}$ from TABLE II into the Eq. (1) starts the iteration against $\varphi=-2$ as explained in Ref. 17. It separates the center of charge from the center of electric moment in the iodate ion and 
gives the direction and magnitude for the polarization vector in it. This is repeated with new atomic charges and stopped until the ratio of the components of second electric moment $\left(\Theta_{x x}^{\prime} /\right.$ $\left.\Theta_{z z}^{\prime}\right)$ reaches the reference value of the ratio of refractive indices $\left(\mathrm{n}_{\mathrm{e}} / \mathrm{n}_{\mathrm{o}}\right)$ as shown in TABLE III.

Eq. (2) is used to calculate the magnitude of the polar vector of first rank in the $\mathrm{IO}_{3}{ }^{-}$ion and the spontaneous polarization, $\boldsymbol{P}_{\boldsymbol{s}}$, in the unit cell with the final charges and iterated coordinates of iodine and oxygens. $\mu_{z}^{\prime}$ is the net value of first electric moment and $q$ is the total charge of the ion.

Eqs. (3)-(5) express the net values of the polar second electric moments in the mutually orthogonal semi-axis directions of the quadric in a hexagonal symmetry. They ought to work in all multinuclear ions regardless of their geometric forms.

\section{Polar vector, $\mu_{z}^{\prime}$, and spontaneous polarization, $\boldsymbol{P}_{\boldsymbol{s}}$.}

The value of the polar vector, $\mu_{z}^{\prime}$, of one iodate ion in the structure description Fig. 1(a) according to Eq. (2): $\mu_{z}^{\prime}=\Sigma e_{i}\left(z_{i}-Z\right)=\mu_{z}-q Z=-4.1217 \cdot 10^{-2}$ and $\mu_{z}^{\prime}$ is pointing in the $-c$-axis direction.

The value of spontaneous polarization, $\boldsymbol{P}_{\boldsymbol{s}}$, with two iodate ions in the unit cell according to Eq. (6).

An example is given for the structure in Fig. 1(a):

$$
\boldsymbol{P}_{s}=2 \cdot \frac{p}{V} \cdot c \cdot \mathrm{e} \cdot 10^{20} \mathrm{Cm}^{-2}
$$

where $p=\mu_{z}{ }^{\prime}, c=5.1725 \AA, \mathrm{e}=-1.6021917 \cdot 10^{-19} \mathrm{C}$ and $V=134,610 \AA^{3}$.

$\boldsymbol{P}_{\boldsymbol{s}}=-\left(2 \cdot \frac{-0.041217}{134,610} \cdot 5.1725 \cdot\left(-1.6021917 \cdot 10^{-19}\right)\right) \cdot 10^{20} \mathrm{Cm}^{-2} \Rightarrow \boldsymbol{P}_{\mathrm{S}}=-5.0751 \cdot 10^{-2} \mathrm{Cm}^{-2}$. $\boldsymbol{P}_{\boldsymbol{s}}$ is pointing in the $-\mathrm{c}$-axis direction. Values of $\mu_{z}{ }^{\prime}$ and $\boldsymbol{P}_{\boldsymbol{s}}$ in the unit cells of four structure descriptions of $\alpha-\mathrm{LiIO}_{3}$ are given in TABLE IV.

TABLE III. The summary of the first two and the final step of the iterations of the $z$-coordinates of iodine and oxygen and the components of second electric moments in the structure description Fig. 1(a) and the final step in the other three structure descriptions in Figs. 1(b)-(d). The initial charges of -0.25 are applied for all atoms in one $\mathrm{IO}_{3}{ }^{-}$ion in each structure description. $\mathrm{x}$ - and $\mathrm{y}$-coordinates are taken directly from the TABLE II. 


\begin{tabular}{|c|c|c|c|c|c|c|c|}
\hline Figure & Atom & Charge & $\mathrm{z}$ & $\Theta^{\prime}{ }_{x x}=\Theta^{\prime}{ }_{y y} \mathrm{e} \AA^{2}$ & $\Theta_{z z}^{\prime} \mathrm{e}^{2}$ & $\Theta^{\prime}{ }_{x x} / \Theta_{z z}^{\prime}$ & $\mathrm{n}_{\mathrm{e}} / \mathrm{n}_{\mathrm{o}}$ \\
\hline $1(\mathrm{a})$ & $\mathrm{I}$ & -0.25 & 0.660568 & & & & \\
\hline & $\mathrm{O}$ & -0.25 & 0.498768 & -0.953706 & -1.221736 & 0.780615 & \\
\hline & $\mathrm{I}$ & -0.15 & 0.678144 & & & & \\
\hline & $\mathrm{O}$ & -0.28333 & 0.516344 & -1.080854 & -1.220005 & 0.885907 & \\
\hline & $\mathrm{I}$ & -0.11524 & 0.684371 & & & & \\
\hline $1(\mathrm{~b})$ & $\mathrm{I}$ & -0.11065 & 0.397624 & & -1.219613 & 0.922480 & 0.922484 \\
\hline & $\mathrm{O}$ & -0.29645 & 0.559424 & -1.130905 & -1.225930 & 0.922487 & 0.922484 \\
\hline $1(\mathrm{c})$ & $\mathrm{I}$ & -0.11065 & 0.397624 & & & & \\
\hline & $\mathrm{O}$ & -0.29645 & 0.559424 & -1.130905 & -1.225930 & 0.922487 & 0.922484 \\
\hline $1(\mathrm{~d})$ & $\mathrm{I}$ & -0.11524 & 0.684371 & & & & \\
\hline & $\mathrm{O}$ & -0.29492 & 0.522571 & -1.125068 & -1.219613 & 0.922480 & 0.922484 \\
\hline
\end{tabular}

\section{Refractive indices and axial vectors of second rank}

The refractive indices are measured with a nonpolar light of the accurate wavelength and in the right-handed crystal the light is traveling in the crystallographic a-axis direction. A mirror image structure to a right-handed crystal is a left-handed enantiomorph with an opposite handedness, where the light propagates in the $b$-axis direction. The semi-axes of the indicatrix are mutually perpendicular in a hexagonal crystal and contain information of the principal optical refractive indices, $n_{o}=n_{o} \neq n_{e}$. The indices, $n_{e}=1.734949$ and $n_{o}=1.880736$, with the ratio $n_{e} / n_{o}=0.922484$, are calculated from the reliable dispersion formulas with $632.8 \mathrm{~nm}$ for $\alpha-\mathrm{LiIO}_{3}$ crystal. [20] The structure description in Fig. 1(b) is right-handed and the corresponding second electric moments from TABLE III are $\Theta_{z z}^{\prime}=-1.225930$ e $\AA^{2}$ and $\Theta_{y y}^{\prime}=$ $-1.130905 \mathrm{e} \AA^{2}$ and the ratio $\Theta_{z z}^{\prime} / \Theta_{y y}^{\prime}=1.084026$. But these two ratios are reciprocals of each other and the only explanation for this is that the measured refractive indices $n_{e}$ and $n_{o}$ must contain information about the axial vectors of second rank formed by vector cross products between a wave vector of light in the $a$-axis direction and the second electric moments derived from the $b$ - and $c$-axis directions, respectively, when a light beam is propagating towards the observer in the $\alpha-\mathrm{LiIO}_{3}$ crystal. With other words the index $n_{o}$ is a measure of the information from an axial vector of second rank on the $a-b$ plane, which arises from the second electric 
moment in the $c$-axis direction of the crystal. The index $n_{e}$ contains information from an axial vector of second rank in the $c$-axis direction, which is generated from the second electric moment in the $b$-axis direction, but deviates $30^{\circ}$ from it on the $a$-b plane of the crystal. The similar inverted relationship between the ratios of the optical refractive indices and the polar vectors of second rank is earlier observed in the hexagonal, polar $\mathrm{LiNaSO}_{4}$ [17] and in the tetragonal, nonpolar $\alpha-\mathrm{TeO}_{2}[21]$ crystals.

The measurements of the refractive indices of the absolute structure description in Fig. 1(b) are illustrated (not in scale) in Figs. 3(a) and 3(b). In the following the rotational character of the axial vector is described in terms how a circularly polarized light will rotate in the vector. $\mathbf{k}_{\mathbf{a}}$ is a wave vector of light and the polar second electric moments, $\Theta_{x x}^{\prime}, \Theta_{y y}^{\prime}$ and $\Theta_{z z}^{\prime}$, denoted by $\mathbf{A}$, $\mathbf{B}$ in the positive directions of the semi-axes of the quadric and $\mathbf{C}$ in the negative $c$-axis direction of the crystal. The obtained axial vectors are indicated by $\mathbf{B}^{\prime}$ and $\mathbf{C}^{\prime}$. Fig. 3(a) is designed to correspond the measurement of the ordinary refractive index, $n_{o}$, in a right-handed crystal, when a light ray propagates in the $a$-axis direction of $\alpha-\mathrm{LiIO}_{3}$ crystal towards the observer, who reads the information from the axial vector, $\mathbf{B}^{\prime}=\mathbf{k}_{\mathbf{a}} \mathbf{x} \mathbf{C}$, (not $\mathbf{B}^{\prime}=\mathbf{C} \times \mathbf{k}_{\mathbf{a}}$, because vector cross products are not commutative). The vectors $\mathbf{k}_{\mathbf{a}}, \mathbf{C}$ and $\mathbf{B}^{\prime}$ follow the right-hand rule and the formed axial vector, B', is dominant and has a clockwise rotation character. According to Fig. $3(\mathrm{~b})$ the crystal is reoriented for reading the information from the extraordinary refractive index, $n_{e}$, against the crystallographic $c$-axis. The axial vector, $\mathbf{C}^{\prime}=\mathbf{k}_{\mathbf{a}}$ $\mathbf{x} \mathbf{B}$, arises from the $\mathbf{A}-\mathbf{B}$ plane of the quadric. The vectors $\mathbf{k}_{\mathbf{a}}, \mathbf{B}$ and $\mathbf{C}^{\prime}$ follow the left-hand rule and the formed axial vector, $\mathbf{C}^{\prime}$, will rotate the circularly polarized wave counterclockwise. Figs. 3(c) and 3(d) present the refractive index measurements in Fig. 1(c), which is an enantiomorphic mirror imagine structure to Fig. 1(b). It is a left-handed crystal, where a light ray travels in the $b$-axis direction. In the Fig. $3(\mathrm{c})$ the axial vector $\mathbf{A}^{\prime}=\mathbf{k}_{\mathbf{b}} \mathbf{x} \mathbf{C}$ is a dominant, where the vectors $\mathbf{k}_{\mathbf{b}}, \mathbf{C}$ and $\mathbf{A}^{\prime}$ follow the left-hand rule and accordingly $\mathbf{A}^{\prime}$ has a counterclockwise rotation character. In Fig. 3(d) $\mathbf{C}^{\prime}=\mathbf{k}_{\mathbf{b}} \mathbf{x} \mathbf{A}$ express that the vectors follow the right-hand rule and $\mathbf{C}^{\prime}$ makes the circularly polarized wave to rotate clockwise. These four Figs. are designed in the right-handed reference coordinate axis system. [22] The dominant components of the formed axial vectors, $\mathbf{B}^{\mathbf{\prime}}$ and $\mathbf{A}^{\mathbf{\prime}}$, have opposite rotational characters and the handedness of them reveal already the optical chirality of the two enantiomorphic crystals of $\alpha-\mathrm{LiIO}_{3}$. 


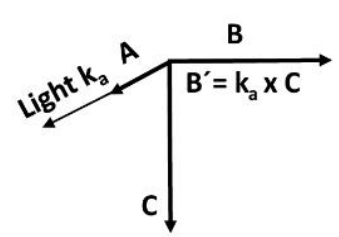

3(a)

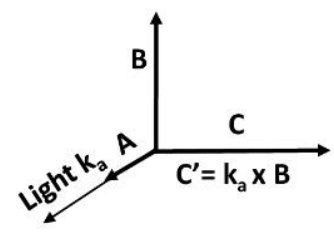

3(b)

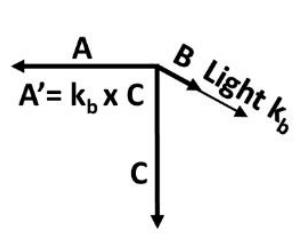

$3(\mathbf{c})$

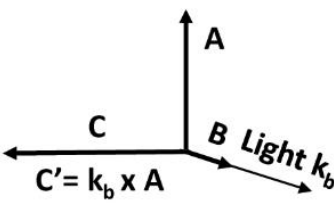

3(d)

Figure 3. Second electric moments, wave vector of light and axial vectors in the two structure descriptions: (3a) and (3b) in Fig.1(b); (3c) and (3d) in Fig. 1(c):

3(a) $\mathbf{B}^{\prime}=\mathbf{k}_{\mathbf{a}} \mathbf{x} \mathbf{C}$ follows the right-hand rule (R) with a clockwise rotation character.

3(b) $\mathbf{C}^{\prime}=\mathbf{k}_{\mathrm{a}} \times \mathbf{B}$ follows the left-hand rule (L) with a counterclockwise rotation character.

3(c) $\mathbf{A}^{\prime}=\mathbf{k}_{\mathbf{b}} \mathbf{x} \mathbf{C}$ follows the left-hand rule (L) with a counterclockwise rotation character.

3(d) $\mathbf{C}^{\prime}=\mathbf{k}_{\mathbf{b}} \mathbf{x} \mathbf{A}$ follows the right-hand rule (R) with a clockwise rotation character.

\section{Gyration tensor components $g_{11,} g_{22}$ and $g_{33}$}

The gyration tensor is an example of an axial tensor of second rank. Its components, $g_{11,} g_{22}$ and $g_{33}$, are axial vectors of second rank. [22] In this study the ratios of the measured refraction indices, of the gyration tensor components, of the axial and polar vectors of second rank and of the refractive indices derived from the axial vectors have the following relationships in the four structure descriptions of $\alpha-\mathrm{LiIO}_{3}$ :

a) Fig. 1(b) and (d): $\mathrm{n}_{\mathrm{e}} / \mathrm{n}_{\mathrm{o}}=\mathrm{g}_{33} / \mathrm{g}_{22}=\mathrm{C}^{\prime} / \mathrm{B}^{\prime}=\left(\mathrm{k}_{\mathrm{a}} \mathrm{x} \mathrm{B}\right) /\left(\mathrm{k}_{\mathrm{a}} \times \mathrm{C}\right)=\mathrm{B} / \mathrm{C}=\Theta_{y y}^{\prime} / \Theta_{z z}^{\prime}=n_{L} / n_{R}$.

b) Fig. 1(c) and (a): $n_{\mathrm{e}} / \mathrm{n}_{\mathrm{o}}=\mathrm{g}_{33} / \mathrm{g}_{11}=\mathrm{C}^{\prime} / \mathrm{A}^{\prime}=\left(\mathrm{k}_{\mathrm{b}} \mathrm{X} \mathrm{A}\right) /\left(\mathrm{k}_{\mathrm{b}} \mathrm{X} \mathrm{C}\right)=\mathrm{A} / \mathrm{C}=\Theta_{x x}^{\prime} / \Theta_{z z}^{\prime}=n_{R} / n_{\mathrm{L}}$.

The following terms can be equalized in magnitudes:

$g_{33}=\Theta^{\prime}{ }_{y y}$ and $g_{22}=\Theta_{z z}^{\prime}$ in Fig. 1(b) and 1(d) and $g_{33}=\Theta_{x x}^{\prime}$ and $g_{11}=\Theta_{z z}^{\prime}$ in Fig. 1(c) and 1(a), but due to symmetry $g_{33}=\Theta_{x x}^{\prime}=\Theta_{y y}^{\prime}$ and $g_{11}=g_{22}=\Theta_{z z}^{\prime}$ in the four structure descriptions, which verify the inverted relationship between the ratios of the optical refractive indices and the polar vectors of second rank in the $\alpha$ - $\mathrm{LiIO}_{3}$ crystal. 
The components of the second electric moment in units $\mathrm{e}^{2}$, in TABLE III, can easily be converted to the principal gyration tensor components in the units coulomb centimeter ${ }^{2}(=$ $\mathrm{Ccm}^{2}$ = buckingham) according to Eqs. (7) and (8):

$$
\begin{aligned}
& g_{11}=g_{22}=\Theta_{z z}^{\prime} \cdot\left(-1.6021917 \cdot 10^{-19}\right) \cdot 10^{16} \mathrm{Ccm}^{2} \\
& g_{33}=\left(\Theta_{x x}^{\prime}=\Theta_{y y}^{\prime}\right) \cdot\left(-1.6021917 \cdot 10^{-19}\right) \cdot 10^{16} \mathrm{Ccm}^{2} .
\end{aligned}
$$

$\alpha-\mathrm{LiIO}_{3}$ belongs to a hexagonal symmetry group 6 . One part about the diagonal of the gyration tensor has three components of which two are equal, $g_{11}=g_{22} \neq g_{33} . g_{11}$ and $g_{22}$ are faster components (dominants) with higher magnitudes than $g_{33}$ in all four structure descriptions of the $\alpha-\mathrm{LiIO}_{3}$ crystal. The values of them for one iodate ion in the four structure descriptions are written in TABLE IV.

\section{Magnitude and sense of optical rotation}

When a plane polarized light enters the uniaxial crystal in the optic axis direction, it splits up into the right-, $n_{R}$, and left-hand, $n_{L}$, circularly polarized waves, which rotate with the same angular velocity in opposite directions according to the theory of optical activity. [22] The waves are in phase at the entrance, but they traverse with slightly different wavelengths depending on the right- and left-handed character of the crystal, which causes a change in phase of the waves when they emerge the crystal. The faster component makes fewer revolutions in the medium and will be ahead in phase. The optical rotation in the crystal will have the same rotational sense as the faster of the two circularly polarized components has at the exit point. ${ }^{22}$

The right-hand circular component arising from the axial vector $\mathbf{B}^{\prime}$ with a clockwise rotational character, is the faster and dominant component in the right-handed structure descriptions of $\alpha$ $\mathrm{LiIO}_{3}$ crystal, in Figs. 1(b) and 1(d), which both have the same structural and optical chirality. The rotation from $\mathbf{B}^{\prime}$ is dominating over the slower component $\mathbf{C}^{\prime}$. The combined rotation is seen as counterclockwise $\left(n_{L}\right)$ in the optic axis direction towards the light source by an observer. The both structure alternatives will be levorotatory. The optical rotation per unit path in the crystal can be calculated according to Eq. (9) as seen by an observer.[22]

$$
\phi=\frac{\pi \cdot\left(n_{L}-n_{R}\right)}{\lambda} \text { radians }
$$


When the rotation is described in the left-handed and enantiomorphic structure alternatives in Figs. $1(\mathrm{a})$ and $1(\mathrm{c})$, the faster component originating from the axial vector $\mathbf{A}^{\prime}$, is left-hand circularly polarized and dominates over the slower component $\mathbf{C}^{\prime}$. The difference between them is seen as a clockwise $\left(n_{R}\right)$ rotation towards the light source by an observer, which makes these two structure descriptions dextrorotatory.

The unit cell of $\alpha-\mathrm{LiIO}_{3}$ has two iodate ions and when the refractive indices and the gyration tensor components are usually expressed for the samples with a thickness of $1 \mathrm{~cm}$ and the optical rotation with a thickness of $1 \mathrm{~mm}$, respectively, the expressions for the rotation of the plane polarized light in an optic axis direction will be for the two structure descriptions in Figs. 1(b) and 1 (c) with $\lambda=6328 \cdot 10^{-8} \mathrm{~cm}$ according to the Eqs. (10) and (11), respectively:

$$
\begin{aligned}
& \phi=\frac{2 \cdot 0.1 \cdot \pi \cdot\left(g_{33}-g_{22}\right)}{\lambda} \mathrm{rad} . \mathrm{mm}^{-1} \\
& \Rightarrow \phi=-1.511708 \mathrm{rad} . \mathrm{mm}^{-1}=-86.61^{\circ} \mathrm{mm}^{-1} . \\
& \phi=\frac{2 \cdot 0.1 \cdot \pi \cdot\left(g_{11}-g_{33}\right)}{\lambda} \mathrm{rad} . \mathrm{mm}^{-1} \\
& \Rightarrow \phi=+1.511708 \mathrm{rad} . \mathrm{mm}^{-1}=+86.61^{\circ} \mathrm{mm}^{-1} .
\end{aligned}
$$

The rotations are described in the right-handed coordinate axis system. [22] The magnitude and sense of optical rotation in the four structure descriptions of $\alpha$ - $\mathrm{LiIO}_{3}$ crystal are summarized in TABLE IV. The orientations of the dominant axial vectors $\mathbf{A}^{\prime}$ and $\mathbf{B}^{\prime}$ (not scaled) are marked with broken lines in the four structure alternatives of $\alpha$ - $\mathrm{LiIO}_{3}$ in Figs. 1(a)$1(d)$.

TABLE IV. Polar vectors, $\mu_{z}^{\prime}$, and gyration tensor components, $g_{11}=g_{22}$ and $g_{33}$ for one and spontaneous polarization, $P_{s}$, and magnitude and sense of optical rotation, $\phi$, for two iodate ions in the unit cells of four different structure descriptions of $\alpha-\mathrm{LiIO}_{3}$.

\begin{tabular}{|c|c|c|c|c|c|c|}
\hline Figure & $\mu_{z}^{\prime} \cdot 10^{-2}$ & $\begin{array}{c}g_{11}=g_{22} \cdot 10^{-3} \\
\left(\mathrm{Ccm}^{2}\right)\end{array}$ & $\begin{array}{c}g_{33} \cdot 10^{-3} \\
\left(\mathrm{Ccm}^{2}\right)\end{array}$ & $\begin{array}{c}\boldsymbol{P}_{s} \cdot 10^{-2} \\
\left(\mathrm{Cm}^{-2}\right)\end{array}$ & $\begin{array}{c}\phi \\
\left(\mathrm{rad} . \mathrm{mm}^{-1}\right)\end{array}$ & $\begin{array}{c}\phi \\
\left({ }^{\circ} \mathrm{mm}^{-1}\right)\end{array}$ \\
\hline $1(\mathrm{a})$ & -4.1217 & 1.954054 & 1.802575 & -5.0751 & +1.504062 & +86.18 \\
\hline $1(\mathrm{~b})$ & -4.1521 & 1.964175 & 1.811927 & -5.1125 & -1.511698 & -86.61 \\
\hline $1(\mathrm{c})$ & -4.1521 & 1.964175 & 1.811927 & -5.1125 & +1.511698 & +86.61 \\
\hline $1(\mathrm{~d})$ & -4.1217 & 1.954054 & 1.802575 & -5.0751 & -1.504062 & -86.18 \\
\hline
\end{tabular}


The experimentally measured value: $\phi=+86.7^{\circ} \mathrm{mm}^{-1}$ in the dextrorotatory crystal Fig. $1(c)^{6}$ and the calculated value from the atomic polarizabilities: $\phi=-87.4^{\circ} \mathrm{mm}^{-1}$ for the levorotatory crystal in Fig. $1(b)^{15}$ with $\lambda=632.8 \mathrm{~nm}$.

\section{Discussion}

Tables III and IV contain calculated values of the polar and optical parameters of $\alpha-\mathrm{LiIO}_{3}$. All four atoms in the polar iodate ion, $\mathrm{IO}_{3}{ }^{-}$, have negative charges. Net atomic charges in the two enantiomorphic pairs are the best fit values and little divergent from each other causing differences in the values of polar vectors, spontaneous polarization and magnitude of optical rotation as a consequence. The polar vectors and spontaneous polarization have the same sense and almost the same negative magnitudes in all four structure alternatives and the sense of them is impossible to change. According to this study both the pyro- and piezoelectric coefficients are negative in the $\alpha-\mathrm{LiIO}_{3}$ crystal, which is observed also in the experimentally measured values in [11].

This study confirms the common view that the more negative end formed by oxygens and the less negative end of iodine of the polar $\mathrm{IO}_{3}{ }^{-}$ion, must point in the positive and in the negative directions of the polar c-axis, respectively, because the negative charge was developed at the positive end on the both enantiomorphic crystals under tensile stress. [11] The two structure descriptions, which form the enantiomorphic pairs of this crystal, must have the apices of the polar iodate ions pointing in the same c-axis direction. The structure alternatives according to Figs. 1(a) and (b) and Figs. 1(c) and (d) can be directly excluded as the enantiomorphic pairs. The structures in Figs 1(a) and 1(d) and in Figs. 1(b) and 1(c) are remaining, but in the former two alternatives the polar groups are pointing in a wrong direction. The calculated values of the optical rotation in the structure description according to Figs. 1(b) and 1(c) are equal and very close to the experimentally measured value given under the TABLE IV. There is very likelihood that the structure description in the Fig. 1(b) is the absolute structure, though the both structural models have not been tested against the total diffraction intensity data set in the refinement. This type of analysis needs an absolute crystal structure to be known as a base for the determination of correct handedness of the axial vectors and the optical and structural chirality in the enantiomorphic crystals. It has been easier to verify the absolute configurations for the two enantiomorphic pairs of $\alpha-\mathrm{LiIO}_{3}$ in this work. According to this study nature favors 
more to build up the enantiomorphic dextro- and levorotatory pairs of $\alpha$ - $\mathrm{LiIO}_{3}$ crystals, which are mirror images as projected in the views Figs. 1(c) and 1(b), respectively. The structure descriptions according to Figs. 1 (a) and (d) are not existing in $\alpha-\mathrm{LiIO}_{3}$ crystal and have not been more closely discussed in this paper.

This work shows that optical rotation can be extracted from the point charge model in the multinuclear ionic solids by an analysis starting from the measured refractive indices. The handedness of the dominant axial vector in an enantiomorphic crystal reveals the optical rotation character of it without any requirement of the experimental test of the optical chirality in advance. The determination requires the accurate values of lattice and structure parameters and four decimal values of the net atomic charges. The iteration indicates that the wavelengthdependent illumination by light affects both the refractive indices and the net atomic charges in the iodate ions as well. The treatment presented in this study by starting from the distribution of charge densities in crystals offers a relatively simple way to calculate very accurate values of electric moments, polar and axial vectors and spontaneous polarization, which can bring more information for better understanding of the origin of physical properties in crystals.

The calculations presented in this study can be carried out with a programable pocket calculator. They are transparent and can be approved and confirmed by any reader, who is interested in scientific computations. Net atomic charges can be iterated only between two atoms within the symmetry related group of atoms of the multinuclear ions in ionic solids. Otherwise, the net atomic charges must be refined against the collected X-ray data set. The refinement program must allow the separated input models of the scattering factors for both the valence and core electrons to be included. That is possible to do with the superior programs MOLLY and XD (http://xd.chem.buffalo.edu). The programs need integer constraints of charges to be incorporated into the multinuclear ions in solids as in the study of $\mathrm{LiNaSO}_{4}$. [17]

\section{Acknowledgement}

The author thanks The University of Uppsala for the offered possibility to make research in physical properties of crystals. 


\section{References}

[1] Flack, H. D. (2003). Helvetica Chimica Acta, Vol. 86, 905-920.

[2] Rosenzweig, A. \& Morosin, B. (1966). Acta Crystallogr. 20, 758-761.

[3] Morosin, B., (1972). Private Communication, quoted as Ref.25 of Bergman, J.G. \& Crane, G. R. (1974) J. Chem. Phys. 60, 2470-2474.

[4] Li Yin-Yuan, Fan Hai-fu \& Chia-Shou-chuan. (1973). Sci. Sin. (Engl. Ed.) 16, 130 - 135.

[5] Svensson, C., Albertsson J., Liminga, R., Kvick, Å.\& Abrahams, S.C. (1983). J. Chem. Phys. 78, 7343-7352.

[6] Stadnicka, K., Glazer, A. M., \& Moxon, J. R. L. (1985) J. Appl. Crystallogr. 18, 237-240.

[7] Abrahams, S. C. (1994). Acta Crystallogr. Sect. A 50, 658-685.

[8] Turner, E. H. (1976). J. Appl. Crystallogr. 9,52.

[9] Liminga R. \& Abrahams, S. C. (1976). J. Appl. Crystallogr. 9, 42-47.

[10] IEEE Standard 176, (1949). Proc. IRE. 37, 1378-1395.

[11] Yang Hua-guang, Zhang Dao-fan, Chen Wan-chun \& Li Yin-Yuan (1989). J. Appl. Crystallogr. 22, 144-149.

[12] Oseen, C. W. (1915). Ann. Phys. (Leipzig), 48, 1-56.

[13] Born, M. (1922). Z. Phys. 8, 390-417.

[14] Ramachandran, G. N. (1951). Proc. Indian Acad.Sci. 33, 217-227.

[15] Devarajan, V. and Glazer, A. M. (1986) Acta Crystallogr. Sect. A 42, 560-569.

[16] Glazer, A. M. \& Stadnicka, K. (1986). J. Appl. Crystallogr. 19, 108-122.

[17] Karppinen, M. (2915). Acta Crystallogr. Sect. B 71, 334-341.

[18] Buckingham, A. D. (1959). Q. Rev. Chem. Soc. 13, 183.

[19] Buckingham, A. D. (1970). Physical Chemistry. An Advanced Treatise. Vol. IV, p.349. New York: Academic Press.

[20] Weber, M. J. (2003) Handbook of Optical Materials. CRS Press LLC. (https://RefractiveIndex.INFO).

[21] Karppinen, M. (2020) J. Appl. Crystallogr. 53, 1252-1256.

[22] Nye, (1972). Physical Properties of Crystals. Oxford: Clarendon Press. 